\title{
X. On integrating and other apparatus for the measurement of mechanical and electrical forces
}

\section{Vernon Boys A.R.S.M.}

To cite this article: C. Vernon Boys A.R.S.M. (1882) X. On integrating and other apparatus for the measurement of mechanical and electrical forces, Philosophical Magazine Series 5, 13:79, 77-95, DOI: $10.1080 / 14786448208627151$

To link to this article: http://dx.doi.org/10.1080/14786448208627151

Published online: 28 Apr 2009.

Submit your article to this journal $₫$

Џ Article views: 3

Q View related articles $\square$ 


\title{
LONDON, EDINBURGH, AND DUBLIN
}

\section{PHILOSOPHICAL MAGAZINE}

\author{
$A N D$ \\ JOURNAL OF SCIENCE. \\ [FIFTH SERIES.] \\ FE $B R U A R Y 1882$.
}

X. On Integrating and other Apparatus for the Measurement of Mechanical and Electrical Forces. By C. Verron BoYs, A.R.S.M., Demonstrator of Pliysics in the Normal School of Science, South Kensington*.

\section{[Plates III., IV.]}

WHEN in February of this year I described my first integrating-machinef before the Physical Society, I felt that, unless the tangent principle could be so applied as to admit of an indefinite growth of the integral, such principle would be useless for practical purposes. In that machine the integral is determined by the position of a cart, and so is limited by the size of the apparatus. Since that time I have devised a variety of methods of applying the tangent principle in which the integral is determined by rotation, and so there is no limit to the extent to which the integral may grow. In the following paper, which is divided into two parts, I have given in the first a description of a variety of integratingmachines, while in the second are some useful applications of the most simple form of integrator described in Part I.

\section{Part I.}

At the present time there seem to be three types of integrating-machines: 1st, those that may be called radius

* Communicated by the Physical Society, having been read at the Meeting on November 26, 1881.

$\dagger$ Phil. Mag. May 1881.

FPhil. Mag. S. 5. Vol. 13. No. 79. Feb. 1882. 
machines, comprising Sang's planimeter, Clerk Maxwell's sphere machine, and Sir James Thomson's disk sphere and cylinder integrator (Ashton and Storey's steam-power meter also comes under this head); 2nd, sine or cosine machines, comprising Amsler's planimeter and mechanical integrator, and the various wind integrators; 3rd, tangent machines, which, so far as I am aware, are represented only by the cart machine already referred to and those that are the subject of this paper. This class of machines depends on the formula for integration, which, in its geometrical application, finds a curve of which the steepness or the tangent of the inclination (i. e. $\frac{d y}{d x}$ ) is equal to the ordinate of the given curve or to the given function. In my former machine a pointer is made to follow a curve, and by so doing causes a rod to be inclined in such a manner that its tangent is equal to the ordinate. There is also a three-wheeled cart; and the plane of its steering-wheel is by simple mechanism kept parallel to this rod; moreover the horizontal component of the cart's motion is equal to that of the pointer. Under these conditions the vertical component of the motion of the cart (or, shortly, its ascent) determines the integral.

I will now show how this principle is applied in a series of machines in which the integral is determined by rotation and not by linear motion, and in which, therefore, the integral may grow indefinitely. At first let us suppose that the cart in my first machine is incapable of vertical motion; then it, in its attempt to move up or down, will push the paper in the opposite direction. If now the paper is wound on a cylinder with its axis beneath the path described by the front wheel of the cart, and if the hind wheels are supported by some other means, then the cylinder will rotate; and the rate of its rotation will be proportional to the ordinate of the given curve, and the amount of its rotation will be the integral required. Now it will at once appear that the cart and the parallel motion are not wanted, and that the inclination of what was the front wheel of the cart, and what may now be called the tangentwheel, may be determined mechanically by the same method that was adopted to give inclination to the rod. Also if, instead of moving the tangent-wheel along the surface of the cylinder, the cylinder be moved longitudinally under the tangent-wheel while its inclination is determined by suitable means, then, as before, the rotation of the cylinder is a measure of the integral.

As the cylinder must necessarily have a finite length, it cannot be caused to move continuously in one direction under 
the tangent-wheel, but must be made to reciprocate. This motion is most readily produced by use of a "mangle-motion," which converts uniform circular into uniform reciprocating motion. Now, when the motion of the cylinder is reversed, so also is the direction of its rotation; and therefore either the action of the tangent-wheel on the cylinder must be reversed, or there must be a reversing-gear between the cylinder and the mechanism employed to count its revolutions. The action of the tangent-wheel on the cylinder is most easily reversed by having two of them mounted in a frame so that they lie in the same plane, but that one is on one side of the cylinder and the other is on the opposite side. The cylinder is made to bear against one during its forward stroke, and against the other during its return stroke-a change readily effected by the mangle-motion. Then the rotation of the cylinder is independent of the direction of its longitudinal motion. When it is preferred to use only one tangent-wheel, the reversal between the cylinder and the counting mechanism can be easily and perfectly produced by using three bevel wheels and a friction-clutch actuated by the mangle-motion or even by the change of motion of the cylinder.

If the cylinder could be made long enough and the ends bent round so as to join one another, then continuous revolution of the whole ring would take the place of the reciprocation of the cylinder, and the integral would be determined by the rotation of the ring round its circular axis. Such a "smoke-ring" can scarcely be made; but an equivalent can be produced without difficulty. Let there be four barrels, each with a concave instead of a convex outline, mounted on a wheel with their axes in one plane, and with some one generating line on each a quadrant of a common circle concentric with the axis of the wheel. Also let the four barrels be geared to one another by bevel wheels (fig. 1, Plate III.). Now let a tangent-wheel be placed inside the common circle so as to touch it at its lowest point; then, if the tangent-wheel lies in the same plane as the circle, revolution of the wheel supporting the barrels will produce no rotation of those barrels ; but if the tangent-wheel is inclined at all, the rotation of the barrels will be directly proportional to the tangent of its inclination, and inversely proportional to the radius of the barrel at the point of contact. This periodical inequality, due to the changing radius of the barrel, may be eliminated by using a second mechanical smoke-ring made of a series of convex barrels mounted on a wheel, with some one generating line of each a portion of a common circle which lies outside instead of inside the barrels. In this, which may be called an outside H 2 


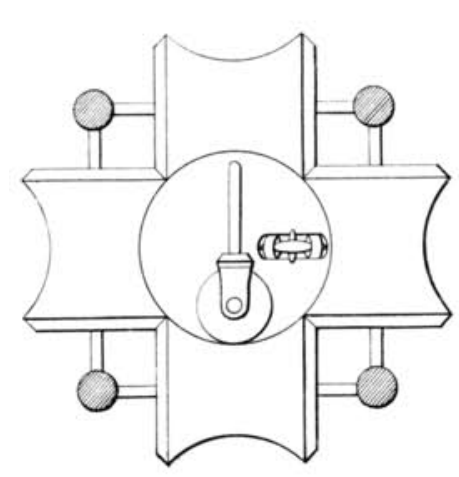

Fig. 1 .

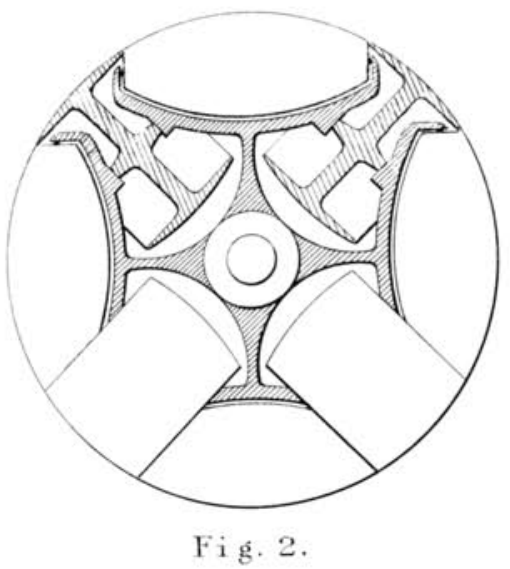

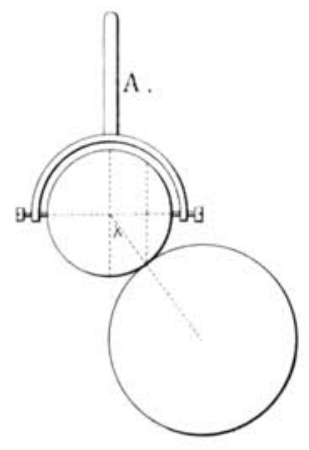

Fig. 3.

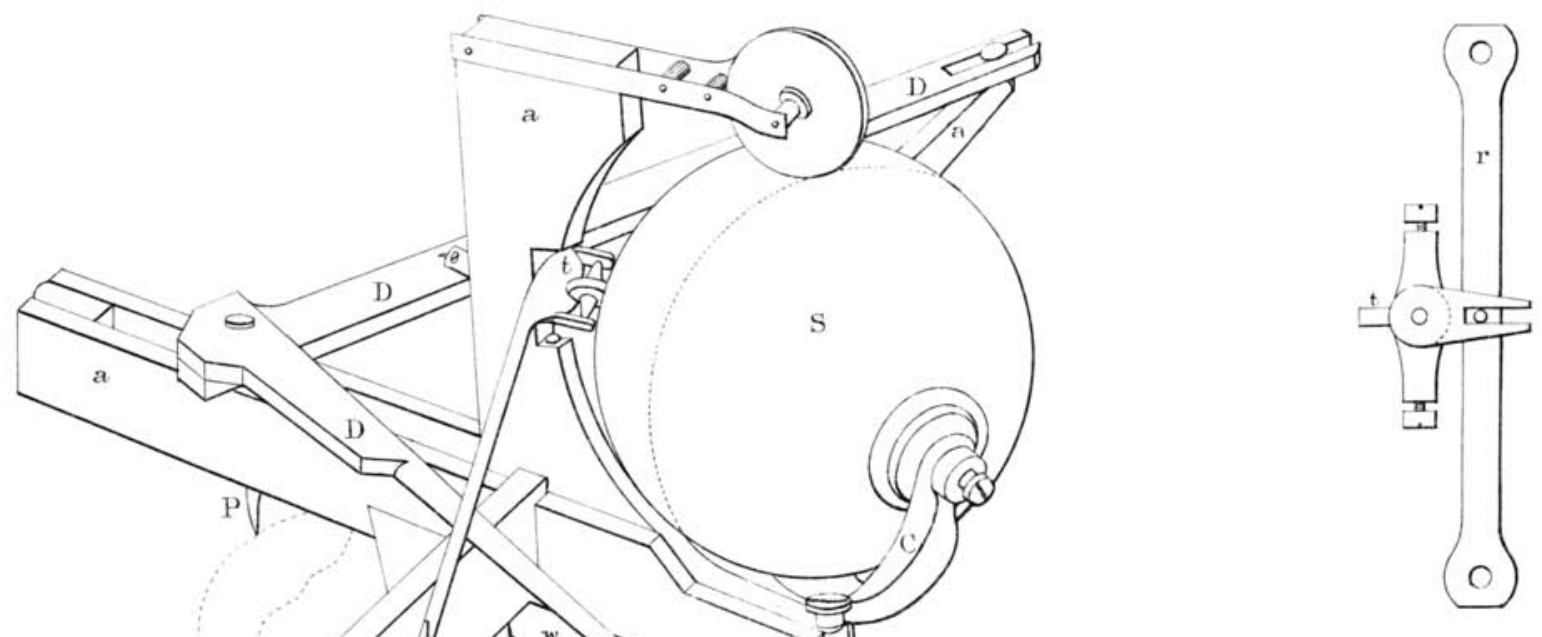

Fig. 7 .
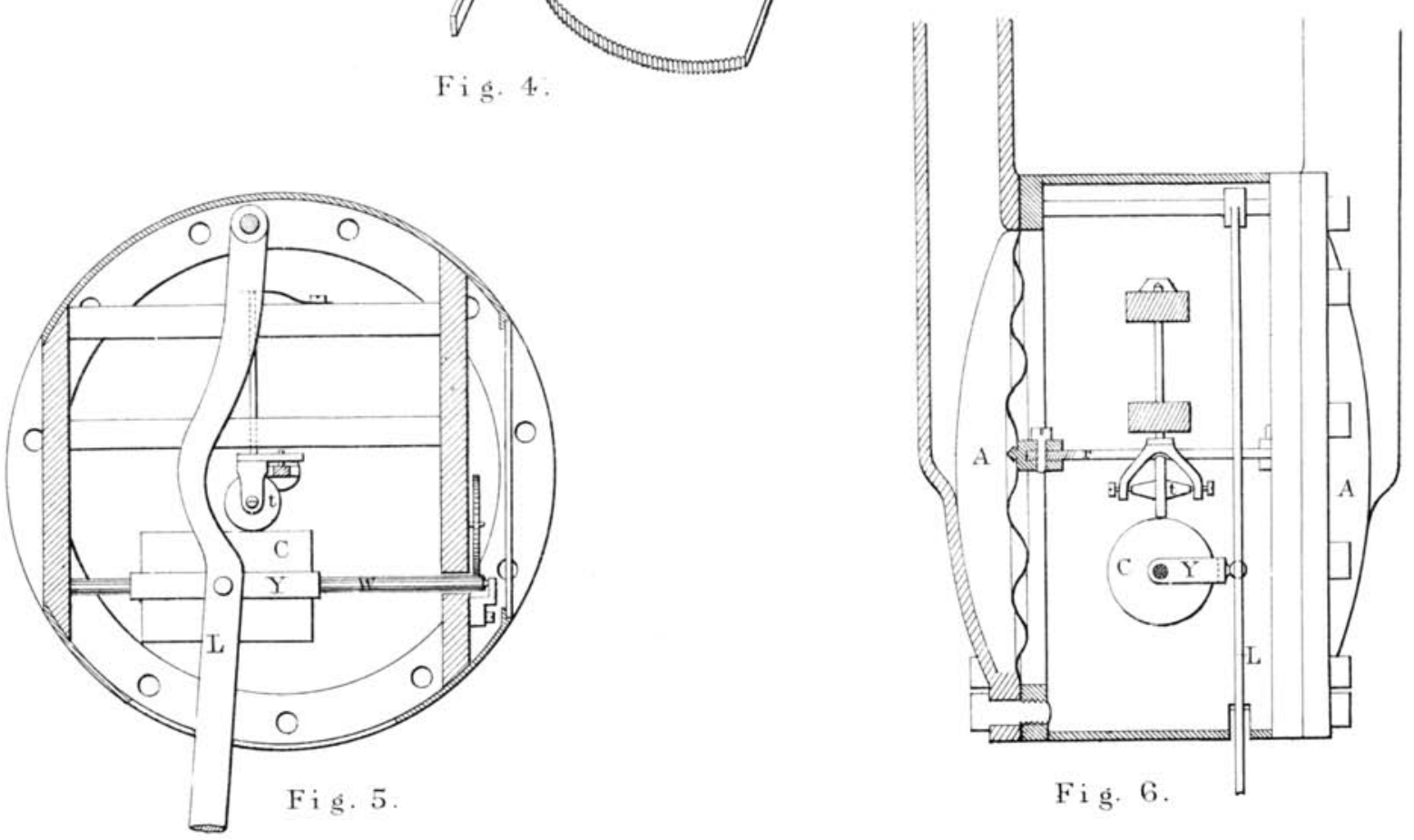
ring, the other being an inside ring, the barrels must not be geared together. Fig. 2 is a plan on a larger scale, partly in section, showing how such barrels might be supported. Now, if this outside ring is placed horizontally within the inside ring and touching it at one point, then revolution without rotation of the inside ring will cause rotation without revolution of the outside ring; if, however, in consequence of the inclination of the tangent-wheel, the barrels of the inside ring are caused to rotate, then such rotation will cause revolu tion of the outside ring; and this revolution will be a true measure of the integral, as the outside ring and the tangent wheel touch barrels of the inside ring at points having the same radius. The astronomical convention with respect to the terms revolution and rotation has been used. By revolution of the ring is meant a turning of the whole round a centre; and by rotation a turning of barrels round their own axes.

Instead of a disk, a sphere similarly mounted may be used for a tangent-wheel, with the same result. Of course the cylinder will be in contact with the sphere at a point on its equator; but if the support of this globe is varied in position, so that the cylinder touches the sphere nearer the poles, then the rate of rotation will depend not only on any former inclination of the plane of the equator of the globe to the axis of the cylinder, but will also be inversely proportional to the cosine of the latitude of the point of contact. The latitude should be brought back to its original value before the rotation of the cylinder is measured. Fig. 3 shows the cylinder in contact with tle sphere at a latitude $\lambda$. It so happens that the radius in the sphere at the point of contact is equal to $\cos \lambda \times$ the radius of the sphere; but this is not the cause of the introduction of that function, as the rotation of the cylinder is independent of the radius of the tangent-wheel. The true reason can be readily discovered by a simple geometrical construction, which, from the length of this paper, I omit. However, a good illustration may be seen by taking a bicycle and causing it to lean over on its side; then a given twist of the handles will be found to produce a greater deviation in the direction of its motion than would be the case if the bicycle were upright. The effect just described is most easily produced by mounting the cylinder on a rocking-frame, so that it can roll round the ball. Though the axis marked $A$ in the figure remains vertical, yet the effect may be considered as due to a leaning to one side of this axis. If, however, the axis $\mathrm{A}$ is caused to lean forwards or backwards, then the rotation of the cylinder, which is still proportional to the tangent of any rotation about $A$, is also proportional to the sine of the 
inclination of $\mathrm{A}$ above the horizon; so that if $\mathrm{A}$ is horizontal, no rotation about $A$ as an axis will produce any effect; but as A rises higher, increased rotation of the cylinder will be produced, the maximum being when $A$ is vertical. As in the former case, so here, the inclination of A should be brought back to its original value before the rotation of the cylinder is measured. This is the method of steering a wheelbarrow when pushing it. The elevation of the handles corresponds to the inclination of A with the horizon; the equality of the elevation of the two handles corresponds to absence of rotation about $\mathrm{A}$; therefore the barrow goes straight. When turning a corner the outer handle is elevated more than the inner one; this corresponds to rotation about $\mathrm{A}$; and the tangent of this rotation, multiplied by the sine of the elevation of the handles, measures the deviation of the barrow from its straight course. This deviation, then, is greater as the elevation of the handles is greater, and therefore in going round a sharp corner the handles should be raised as much as possible. By the means above described, either the quotient or the product of two functions may be directly integrated.

As in my former integrating-machine, so with these, the reciprocal of a function may be integrated by first inclining the tangent-wheel through a right angle; then, when a function passes through 0 from + to - , the tangent-wheel describes on the surface of the cylinder a cusp showing a momentary infinite motion. A machine so arranged may be used to integrate, not the reciprocal, but the function itself, if, instead of moving the eylinder longitudinally, it is caused to rotate, when the longitudinal motion, or the number of reciprocations if suitable gearing is employed, will measure the integral. In a similar way the machine as first described will integrate a reciprocal.

If for any purpose, in addition to the total result, the integral up to any time is required, a diagram must be drawn. This can be effected by covering the cylinder with a layer of black tracing-paper, and allowing a band of paper as wide as the cylinder is long to pass between the tangent-wheel and the black surface. The length of paper passed through the machine represents the integral; and the curve drawn shows its rate of growth continuously. Should it only be required to know the amount of growth during each of a series of short intervals of time, a narrow band (which is more manageable) may be used wrapped round a small wheel at the end of the cylinder, and so arranged that at the end of each double stroke of the cylinder it is caused to bear against the point of a stationary pencil; then the pencil-marks represent equal intervals 
of time, while the distances between them measure the average rate of growth over each interval.

I have at present supposed that the integrating surface is cylindrical; but other surfaces of revolution may be employed for particular purposes. As the rotation of the cylinder depends on the linear motion of its surface, it is clear that its rotation must bo inversely proportional to its diameter. If, therefore, instead of a cylinder any other surface of revolution is taken, its rate of rotation will depend not only on the inclination of the tangent-wheel, but also on the radius of contact. The simplest case is that of a disk with the tangent-wheel mounted so as to be capable of radial movement. Then, if the tangentwheel moves in the direction of its own plane, it will simply describe on the disk a radial line, and there will be no rotation; but if it is inclined at any given angle the disk will rotate, and the rate of its rotation will be proportional to $\frac{1}{r}$, and its whole rotation will be $\int \frac{1}{r} d r$, which is $\log r$. Now the tangent-wheel, in its movement outwards, describes on the surface of the disk a spiral which everywhere cuts the radii at the same angle; therefore in such a spiral the angles are the logarithms of the radii; $i . e$. it is the logarithmic spiral If the inclination of the tangent-wheel is made to depend on some function, then such double-disk machine would integrate $\frac{\phi x}{c+x} d x$, in which $c$ is the radius of contact when $x=0$.

If the axis of the tangent-wheel is made to pass through a fixed point over the disk removed from its line of travel by a right angle, then the tangent of its inclination to the direction of its motion is proportional to the radius of contact: but, other things being equal, the rotation of the disk is inversely as the radius of contact; therefore the amount of rotation of the disk for a given movement of the tangent-wheel is independent of the radius of contact, and the curve traced out on the disk is the spiral of Archimedes. But if, instead of passing over a disk, the tangent-wheel similarly mounted is made to pass along the surface of a cylinder, then the speed of rotation of the cylinder will be proportional to the distance of the disk from its neatral position, and its whole rotation will be $\int c x d x$, or $\frac{c}{2} x^{2}$, and the curve described on the cylinder will be a parabola. This arrangement of the disk and cylinder may be used, as described on page 84 , in a polar planimeter to illustrate the formula $\iint r d r d \theta$. 
After the cylinder and disk, the most simple form for an integrating surface is that of a sphere. Let a sphere be supported, with its axis horizontal, on a frame which can be made to reciprocate about a vertical axis which would, if continued, pass through the centre of the sphere; then, if a tangent-wheel is fixed so as to lie, when its inclination is nothing, in the horizontal plane which passes through the axis of the sphere, angular reciprocation, which must be less than $180^{\circ}$, will cause the tangent-wheel to describe on the sphere a meridian when it is in its neutral position, or a rhumb line if inclined at a constant angle. As the speed of rotation of the sphere is inversely proportional to the radius of contact-that is, to the cosine of the latitude of the point of contact-some means must be adopted whereby the rotation recorded is less than the rotation of the sphere in the same ratio. The most simple plan is to use Amsler's principle, and mount a small sliding and rolling wheel so as to be in contact with the sphere at the highest point on the equator $\left(i\right.$. e. $90^{\circ}$ from the tangent-wheel), but with its plane passing through the centre of the tangent-wheel ; then the rotation of the Amsler wheel is always less than the rotation of the sphere, in the same ratio that the rotation of the sphere is too great. Instead of an Amsler wheel, a cylinder capable of moving longitudinally on its horizontal axis, and in contact with the sphere at a point exactly opposite to the tangent-wheel, would, by pure rolling and without any sliding, take off the correct proportion of motion, since it and the tangent-wheel always touch the sphere at points having the same radius.

Fig. 4 is a perspective view of a polar planimeter in which the integration is effected by a disk sphere and Amsler wheel, as described. All the parts marked $a$ belong to a rigid frame, which balances on and can turn about a vertical spindle, the top of which is just visible below the tangent-wheel $t$. The vertical spindle is fastened to the stationary wheel $w$, which rests on three feet. The segmental wheel $W$ in gear with $w$ is secured to a vertical spindle, the upper end of which carries the crutch C. Screws in the crutch form the horizontal axis about which the sphere $\mathrm{S}$ may rotate. The tangent-wheel $t$ is mounted in a frame which can be turned about a horizontal axis $e$ by means of a lever $l$. The Amsler wheel rests by its weight on the bighest point of the equator of the sphere, which is shown dotted. D D is an L-shaped piece, which carries at the angle the pointer $P$. At the end of the long limb is a slot embracing a pin, as shown. A part of the short limb is made cylindrical; against this part rests the edge of the lever $l$. This edge is not truly radial, but is 
laterally displaced from the radial position to an extent equal to the radius of the cylindrical part of $D D$. This causes the true radius, which is parallel to the axis of the tangent-wheel, to intersect the axis of the cylinder. Now, if the pointer $P$ is moved radially in the slot prepared for it, it is clear that the tangent of the inclination of the tangent-wheel $t$ will be proportional to the square of the distance of $\mathrm{P}$ from the vertical axis about which the machine can turn, also that, during any turning of the machine about this axis, the sphere will turn about its vertical axis at a proportionate speed. Now it has been shown that, when the sphere is made to turn about its vertical axis, the rate of rotation of the Amsler wheel is proportional to such rotation multiplied by the tangent of the inclination of the tangent-wheel-that is, in this case to $r^{2} d \theta$. Therefore the whole rotation of the Amsler wheel is a measure of $\int r^{2} d \theta$; and so, if the pointer $\mathrm{P}$ is taken round any closed curve, the area of that curve may be read off from the Amsler wheel. The wheel $\mathrm{W}$ is three times the radius of $w$; so that the pointer may, if necessary, be taken completely round the pole, and yet the tangent-wheel will only move $120^{\circ}$ on the sphere in latitude. The diameter of the Amsler wheel is one third of that of the sphere, so as to restore the diminished speed. Unlike Amsler's planimeter, this one shows the increment of area for each part of a closed curve, the reason being that it is an exact mechanical equivalent of the polar formula for integration. Though the machine works very well, it cannot be compared to Amsler's as a practically convenient instrument.

An exact mechanical equivalent of the formula $\iint r d r d \theta$ would be produced by retaining all the last machine, except the short limb of the L-shaped piece D D, and mounting on the long limb a tangent-wheel to traverse a cylinder, the rate of rotation of which for a given radial movement of the pointer would be proportional to the distance of the pointer from the pole-that is, to $r d r$-and the whole rotation would be $\int r d r$. Now, if the cylinder were by its rotation caused to change the inclination of the lever $l$ so that the tangent of the inclination of $l$ was proportional to the whole rotation of the cylinder, then, when the pointer was taken round a curve, the rotation of the Amsler wheel would be $\iint r d r d \theta$. In either case, instead of an Amsler wheel, a cylinder mounted as described on the last page would give the integral. 


\section{PART II.}

The practical value of the tangent principle depends on the fact, that the only operation required of the function to be integrated is that of turning more or less a spindle and tangentwheel, which may be as light and delicate as any part of a watch, and of which the moment of inertia may be inappreciable. This is in marked contrast to what is necessary in radius machines: the friction in the common double disk or disk and cone integrator, or the inertia of the ball in Sir James Thomson's machine, would be quite sufficient to make the former useless for the integration of such delicate forces as depend on the actions of electricity, or the latter inapplicable to machinery in rapid movement. Another point about tangent machines is, that the whole process of integration is the result of pure rolling, and any doubt that may be felt as to the effect of the sliding action on the accuracy of cosine machines is here removed. The rest of this paper describes some applications of the disk-cylinder integrator some of which are likely to be of practical value.

\section{Engine-power Meter.}

As work is motion multiplied by pressure, the work done in an engine may be found by integrating the difference of pressure on the two sides of the piston with respect to the motion of the piston. For any one stroke, this is usually done by measuring the areas of the indicator-diagrams, one taken at each end of the cylinder, and repeating, so as to get an average value. But as the work done, or the area of the diagram, is subject to variation depending on the load, pressure, and speed, only guesses can be made as to the whole amount of work that has been done by an engine during any length of time. Any machine, therefore, that will automatically find the total work done should be of value, not only to users of engine-power, but especially to experimentalists who are engaged on testing the efficiency of engines, and on other subjects where total work done should be known. It is only fair to mention that Messrs. Ashton and Story have an enginepower meter in which the integration is effected by a doubledisk integrator acting on the radius principle; but it necessarily suffers from the defects common to all radius machines. The disk-and-cylinder is especially applicable to this particular case; for it is only necessary to make the cylinder reciprocate with the piston of the engine, the motion being of course reduced to a convenient amount, and to make the tangent of tho inclination of the disk vary with the difference in pressure on 
the two sides of the piston of the engine. Then, at any moment, the cylinder will turn with a speed which is proportional to the rate at which work is being done, and the number of revolutions, as measured by a counter, will be a measure of the work done in foot-pounds or other units during any time. Figs. 5 and 6 are views of an engine-power meter, each partly in section. A, A are two boxes with flexible covers, like the corrugated plate in an aneroid barometer. They may bo filled with a mixture of glycerine and water or other liquid, and connected each with one end of the cylinder of the engine. Each diaphragm will feel the pressure, but not the heat, of the steam or gas in the cylinder. The two diaphragms are connected by the rod $r$; and so the effective force tending to bend the diaphragms is the difference of pressure at the two ends of the cylinder. This is the force acting on the piston of the engine. Inclination is given to the tangent-wheel $t$ by the rod $r$ by a pin working in a radial slot, as is better shown in fig. 7. This arrangement causes the tangent of the inclination of the tangent-wheel to be proportional to the displacement of the rod $r$, and so to the force acting on the piston. $\mathrm{C}$ is the integrating-cylinder, which is capable of sliding along, but of turning with a wire $W$, which may be grooved or polygonal; pinion-wire is very suitable. The integrating-cylinder is caused to reciprocate, by means of a yoke $Y$ and lever $L$, in time with the piston of the engine. The stroke is reduced to a convenient amount by attaching a string from the pistonrod to a suitable part of the lever L. Now, as the rate at which the cylinder turns is proportional to the longitudinal motion of the integrating-cylinder multiplied by the tangent of the inclination of the tangent-wheel, and as this is proportional to the motion of the piston multiplied by the force urging it, $i$. e. to the work being done, the whole number of the revolutions of the cylinder will measure the whole amount of work done. If the instrument gets out of adjustment so that the tangent-wheel is not parallel to the axis of the cylinder when there is no force, then whatever error it makes in a forward stroke it will take off in the return stroke; so that no accumulating error will be produced. The diaphragms may either be made of steel or highly elastic metal, in which case they form their own springs; or a softer metal, controlled by an external-spring, might be used. If a diagram is required, one may be drawn as described on page 81 . But it will not be a diagram such as is drawn by an ordinary indicator, but the integral curve of such a diagram; so that force, instead of being represented by the length of an ordinate, will be represented by steepness. Instead of diaphragms as described, 
spring pistons or Bourdon pressure-gauge tubes might be employed to give inclination to the tangent-wheel.

\section{Integrating Dynamometers.}

The disk-cylinder integrator may be applied to measure the whole amount of work transmitted by shafting or belting. In the case of shafting, what is called a differential coupling - that is, a contrivance which transmits any motion, but measures the force causing such motion-is employed to give inclination to the tangent-wheel. In the case of belting, any of the known dynamometers may be employed for the same purpose; while a mangle-motion driven by the revolving shaft or travelling band, causes the cylinder to reciprocate. Either of the methods given on page 79 may be employed to produce continuous growth of the integral in one direction. As the work transmitted at any moment is force multiplied by motion, and as the tangent of the inclination of the tangent-wheel is proportional to the force, while the reciprocating motion of the cylinder is proportional to the motion, the rate of revolution of the integrating cylinder will be proportional to the rate at which work is being done, and the whole number of revolutions will give the whole amount of work done. If at any time the force causing the motion should change sign and so resist it, as is the case in an engine when there is much cushioning, then the tangent-wheel will incline the other way and take off from the record a corresponding amount of work.

\section{Electric-Current Meters.}

The application of the disk-cylinder integrator to an electric-current meter is very obvious. Figs. 8 and 9 are two views of an electric meter, in which the inclination of the magnet $M$ is effected by the electric current passing in a large coil surrounding the instrument. The magnet $\mathrm{M}$ and the tangentwheel $t$ are each fixed on the same spindle, which is vertical, and which is very light and delicate. The weight of the magnet produces the necessary pressure between the tangentwheel and the integrating-cylinder $\mathrm{C}$; and as the surface of each is convex, the friction resisting the turning of the tangent-wheel by the magnet is very small. The cylinder is supported in a bell-crank frame $F$, which can be made to reciprocate along the wire $\mathrm{W}$ by means of the mangle-motion $\mathrm{m} \mathrm{m}$. The mangle-motion is actuated by clockwork, which may be wound by the current itself when necessary, should such a course be desirable. Fig. 10 shows the construction of a suitable mangle-motion. The pinion can turn, but not move otherwise, 
while the frame carrying the racks can move either longitudinally or laterally. A pin projecting centrally from the pinion enters the slot, which is shaded in the figure, and so causes the pinion to gear with the two racks alternately. This lateral movement of the rack-frame is made use of to depress the integrating-cylinder during its back stroke, at which time the magnet rests on the shoulder $\mathrm{S}$. As the tangent of the inclination of the tangent-wheel is proportional to the currentstrength, and as the rate of rotation of the cylinder is proportional to the tangent of the inclination of the tangent-wheel, the cylinder will turn with a speed which is proportional to the current-strength, and the whole number of turns, as shown by the counter, will be a measure of the quantity of electricity that has passed. In the arrangement described, time is divided into a great number of equal intervals, and the current-strength during each alternate one considered. After any considerable time, such a sampling of the current would give just as exact a result as would be obtained by integrating continuously. A quick-return mangle-motion might be employed to diminish the proportion of ineffective time; or the whole time could be made effective by keeping the cylinder in continuous contact, and actuating a reversing-gear between the cylinder and a counter by means of the lateral movement of the manglemotion. It would be well to employ a catch on the armature of a subsidiary electromagnet, so as to stop the clockwork, except when a current is passing. This current-meter, like Edison's electrolytic meter, is a direction-meter. If the current is passing in one direction, it counts it positive ; if in the opposite direction, it counts it negative. A reverser actuated by a polarized armature could be employed to make the meter count as positive, a current passing either way, and so make it applicable to the case of alternating currents. Another kind of current-meter, which is by its nature independent of the direction of the current, would be preferable to the magnetic-needle meter and reverser combined.

Figs. 11 and 12 represent an electric-energy meter, which will be described later; but they will serve as diagrams to illustrate a description of the second current-meter. $\mathrm{mm}$ is the mangle-motion, which causes the cylinder $\mathrm{C}$ to reciprocate and bear alternately against the two tangent-wheels $t t$. These wheels are mounted in a common swivelling-frame, which ordinarily is kept vertical by the weights X X, but which may be inclined by a force due to any cause tending to turn the beam B. Now the turning-power of the weights $\mathrm{XX}$ varies as the sine of the inclination; while the turningpower of a force acting downwards applied to the point $p$ varies 
as the cosine of the inclination; therefore the beam will set itself at such an angle that the tangent of the inclination is proportional to the force. If, therefore, the point $p$ of the beam can be pulled downwards by a force which is proportional to the strength of the current, an electric meter will be the result. The coils shown in the diagrams, which belong to the energy-meter, must be removed and replaced by an electromagnet and armature of peculiar construction. Let there be an electromagnet with pole-pieces a certain distance apart, and let there be between them a wedge of iron at its narrow end increasing in thickness or width rapidly, and towards its thicker parts much more slowly; then, on moving such a wedge forwards between the poles, but without touching them, it will at first facilitate by its movement magnetic induction at a great rate; and as it fills up the space, even though the induction through it is greater, yet the increase of that induction is less. Now, as the rate at which magnetic induction is increased by movement measures the force with which such a wedge is pulled forwards, the wedge will, if suitably formed, experience a force with a given current-strength which is less as its entrance is greater, except over a small space near its starting position, where the force should, if possible, be infinite. Also, if the wedge is fixed in position and the current made to vary, it will, so long as the magnetic limit is not approached, experience a force which varies as the square of the current; therefore, if the motion of the wedge is resisted externally by a force which varies as its displacement, it will enter to such an extent that the amount of its entrance is proportional to the current. Let such a wedge be carried by the beam $B$, so that when it is at its zero position the beam is horizontal; then the inclination of the beam will be greater when the current is greater, and, except with very weak currents, may be made to vary so that its tangent is proportional to the current. The same principle might be applied in another way by causing rotation instead of linear movement of the armature to facilitate induction, and by resisting such motion by a pendulum-weight as before, or by a hair-spring. The armature then would have to be shaped something like an $\mathrm{S}$, and adjusted experimentally so as to give, except with very small currents, a deflection whose tangent is proportional to the current. In Sprague's or Edison's electrolytic meters (the only ones of which I have heard which can lay any claim to being called current-meters at all) a portion only of the current is sent through the meter, and the rest passed by in a shunt. Now, if it were certain that the same proportion of the whole current always passed the meter, there would be 
no objection to such a course; but as a rise in temperature makes an electrolyte a better, and a metal a worse conductor, any electrolytic meter combined with a shunt would have a tendency to show too much in warm weather or if warmed by the current. If, again, there is any polarization, and that polarization is not strictly proportional to the current, then another error will be introduced. It would seem therefore necessary, if accurate indications are required, to make the whole current pass through an electrolytic meter.

\section{Electric-Energy Meters.}

Since the energy expended by an electric current between any two points is equal to the current multiplied by the difference of potential of those points-that is, to the main current multiplied by a shunt current passing in a wire of high resistance between the two points-an electric-energy meter may be made by combining two electric-current meters, which take account of the direction in which the electricity passes, in such a way that the first integrates the main-current strength, and that the mangle-motion of the second is driven by the integrating-cylinder of the first: then, if the needle of the second is deflected by the shunt current, the rotation of the second cylinder will give the integral of the energy expended; This is obvious; for the rate of rotation of the second cylinder is proportional to its rate of reciprocation multiplied by the tangent of the inclination of its tangent-wheel-that is, to the strength of the current in the first machine multiplied by the strength of the current in the second; so its rate of turning is proportional to the rate at which energy is being expended, and its whole rotation is a measure of the total energy. If at any time either the main or the derived current, but not both, changes sign, then the second cylinder will begin to turn the other way, showing that the current is not doing work in the portion of the conductor between the points, but is being caused to flow by an action of some kind taking place in that portion. If both currents change sign, then, as before, work is being done; and though the first machine is working backwards, the second is working forwards. A combination of two machines, as described, would integrate both the current and the energy. A more simple and practicable machine for integrating energy alone is shown in figs. 11 and 12 . The integrating mechanism has been already described; the electrical principles employed must now be explained. If two wires, one conveying the main current and the other the derived current, are near one another, they will attract or repel one another with a force which is proportional to the product 
of the two currents-that is, to the energy being expended; but if the wires are allowed to move, the force will depend also on the position. The question then is, how can the wires be so arranged that the force exerted may be used to incline the tangent-wheels and yet be independent of their motion. The arrangement of solenoids shown in section in fig. 12 is a complete and perfect answer. $S_{1}$ and $S_{2}$ are two fixed solenoids concentric with one another; and the main current is made to pass through each in the same direction. $S_{3}$ is a solenoid made of a very great length of fine wire, preferably of aluminium silver, as suggested by $\mathrm{Mr}$. Imray; and the upper half is wound in one direction and the lower half in the opposite direction; and the derived current is made to pass through it so as to pass in the upper half in the same direction as the main current passes in the fixed solenoids, and in the lower half in the opposite direction. This solenoid is hung in the annular space between the fixed ones by a band passing over the arc of the beam $B$. The tubes within and without the fixed solenoids and the rings above and below them, all of which are shown dark in the figure, are made of iron, and may or may not be used according as the currents employed are in general weak or strong. But whether the tubes are or are not retained, the use of the rings has certain advantages, which I now proceed to explain. Fig. 13 is a diagram taken by iron filings, which shows the distribution of the lines of force due to the fixed solenoids alone, without the tubes or rings. Here the lines of force cut the walls of the solenoid at an oblique angle, so that the force, which is at right angles both to the wires and the lines of force, tends to stretch the movable coil, and only a portion of it is effective in dragging down the solenoid : moreover the lines of force are very widely distributed over the solenoid, so that, unless it is of very great length, the upper part will leave by its motion many lines. It is true that on the lower end the movable solenoid will, when at its central position, enter as many lines in an element of motion as it leaves at its upper end; but after any considerable movement the upper end will leave many more than the lower end enters ; and the force, as it is proportional to the number of lines enclosed, will become less as the solenoid moves from its central position. This will be referred to again later. Now the iron rings act as traps, so to speak, and catch nearly all the lines of force which without them stray over so great a space. Fig. 14 shows the field produced when the iron is present. It will be seen at once that nearly the whole of the induction takes place across a very narrow band of the solenoid, and that the lines of force, 
where they cut the solenoid, are nearly horizontal; so that practically the whole of the force developed tends to drag the solenoid downwards, instead of being partly spent, as before, in producing a stretching strain. The consequence is that the movable solenoid may be made very much shorter than would be necessary if no rings were present. The tubes, having a higher coefficient of induction than air, merely serve to increase the number of lines of force, rather than affect their distribution.

If a movable solenoid, arranged as described, is hung by a band passing over the arc of the beam $\mathrm{B}$, then the turning moment due to a down-pulling force is, within certain limits, constant, while the force restraining motion varies as the sine of the inclination of the beam; but had it been hung from the point $p$, the turning moment would have been proportional to the cosine of the inclination, and the tangent of the inclination would have measured the force. What is wanted is to make the tangent of the inclination proportional to the product of the two currents; and, as explained, this could be attained by using a long solenoid hung from the point $p$. But such an arrangement causes a double inconvenience; for not only is a long solenoid itself inconvenient, but the fact that it is hung from an arm and not from an arc canses a lateral shifting of the solenoid when the beam $B$ is inclined, thus necessitating a wider annular space for it to pass through. Now the use of a comparatively short solenoid hanging from an arc introduces two errors which are almost absolutely equal and opposite. The error due to the arc is an increase of moment in the ratio of the cosine of the inclination of the beam to 1 ; that is, the error is equal to the versed sine of the inclination ; and, like a thing that increases as the square of a quantity, it is at first quite inappreciable, and it increases in amount with increasing speed as the quantity grows. Now the error due to a short solenoid, such as shown in the figure, is at first nothing; for a given movement will cause the solenoid to enter as many lines of force at one end as it leaves at the other, but as it gets displaced it enters rather fewer than it leaves; and this difference in the number of lines of force increases in amount with increasing speed. Now, if the proportions are so taken that when the inclination of the beam is a little less than the greatest amount permitted to it the actual magnetic error is equal and opposite to the versed-sine error, then, since each is a quantity which grows according to the same kind of law, those errors will be always approximately equal, and their differences very small compared with the errors, and absolutely inappreciable in comparison with the 
quantities of which the errors themselves are small. The geometrical representation (fig. 15) makes this clearer. The two sets of errors may be considered as ordinates of two curves drawn to the same abscissa. The point $p$, where the curves intersect, corresponds to that inclination at which the two errors are made equal, and the origin $\mathrm{O}$ to the central position. Since both errors are of a kind which are inappreciable at first, the two curves will have the axis of $x$ as a tangent; and since the two errors are of a kind which grow in the same kind of way, i.e. at first slowly, and at an increasing rate as they grow, the curves must be nearly similar; and since they have the common point $p$, they must be nearly identical. The distance between the curves at any part gives the actual error there; and this being small in comparison with the ordinates at that part, is inappreciable in comparison with the whole distance of that part from the line $z z$. In the particular case the ordinate $p m$ is about 4 per cent. of $p Q$; so the actual error may be safely neglected. But, finally, should there be any error that can be detected at all, such error may be almost entirely eliminated by putting a few extra turns of wire near the ends or the middle of each half of the movable solenoid, according as the curve of the magnetic error between $O$ and $p$ is below or above the curve of the versed-sine error.

Assuming the truth of what is stated in the last paragraph, we find the tangent of the inclination proportional to the rate at which energy is being expended. But the speed of rotation of the cylinder is proportional to the tangent of the inclination; therefore the number of turns of the cylinder, given by the counting-mechanism in the box over the cylinder, is a measure of the total energy expended. As in the other energymeter, so in this, if the electric current is helped at any time instead of being used, then the integrating-cylinder will turn the other way, and take off from the record an amount_which is a measure of the work expended on the current.

There is a point about the solenoid energy-meter which is worthy of notice. The movable solenoid has an equal number of turns in opposite directions; so it is independent of the magnetic field in which the instrument is placed, and so this meter may be used in workshops or near dynamo-machines without its action being interfered with. For the same reason, when used in houses as gas-meters are for gas, it will be impossible for the householder to tamper with its indications by placing magnets round the instrument.

When very powerful currents are employed, it is well to shunt a certain proportion of the main current past the meter, or, when the electromotive force is very great, to introduce Phil. Mag. S. 5. Vol. 13. No. 79. Feb. 1882. 
into the secondary circuit resistance-coils. To prevent wasto of clockwork energy or of electricity, the main current is made to pass round a subsidiary electromagnet whose armature allows the clockwork to go only when the main current is passing. This armature also, on being attracted, completes the secondary circuit, so that the derived current can only flow when the main current is passing.

The meter may be used as an energy measurer or indicator (not meter) with advantage when setting the carbons of an electric arc. If the two poles are made to touch, though the current is enormously increased, the energy is reduced, owing to a greater falling-off in the difference of potential between the poles. As the carbons are gradually separated the current diminishes, but the difference of potential increases in a higher ratio; so their product increases; that is, the energy expended, and so the heat and light produced, increases. This increase is shown by an increased inclination of the beam. After a time a point is reached at which the current decreases in the same ratio that the difference of potential increases; at this point the inclination of the beam attains a maximum; beyond this the decrease of the current is in a higher ratio than the increase in the difference of potential, so the energy, heat, and light fall off, as is indicated by the diminution of the inclination of the beam. If, therefore, the carbons are placed so that the inclination of the beam is a maximum, then the best effect is being obtained. In the same way, if the current is being employed to drive a machine, the most effective speed for that machine may be found by observing at what speed the inclination of the beam is greatest.

The various meters described depend for their numerical results on two things-(1) the horizontal intensity of the earth's magnetism, (2) the force of gravity. The indications of the first current-meter are inversely proportional to the horizontal intensity, and of the first energy-meter to the square of the horizontal intensity. Each of them is disturbed by changes in the direction of the earth's magnetism.

The second current-meter and the second energy-meter are independent of the magnetic field altogether. Their indications vary inversely as $g$ when the clockwork is regulated by a balance-wheel, or inversely as $\sqrt{g}$ when a pendulum-clock is used. This dependence on gravity is a point of very great importance; for over any one country gravity does not change appreciably, nor does it matter in what direction the machine is placed so long as it is level. By screwing the weights X X up or down, so as to decrease or increase the influence of gravity on the meter, its indications may be regulated to a 
standard measure. Therefore, in making the coils, there is no necessity to count the number of turns exactly, or to lay them with the utmost accuracy: they may be wound in the ordinary way, and then a hundred machines or more connected together, with the main circuits in series and with the derived circuits in series, and a current sent from a suitable source through each series; then, if there is one meter which has been standardized by careful experiment, all the rest can be regulated, just as clocks are, by screwing down the weights $\mathbf{X} X$ of those that are going fast, or screwing up the weights of those that are going slow.

If in the foregoing paper any of the apparatus is not as fully described as it might be, I must plead as an excuse an endeavour to occupy a reasonable space with an account of what is essentially one invention.

XI. The Experimental Comparison of Coefficients of Induction. By Herbert Barfield, B.Sc.*

F all electrical investigations, the experimental comparison of coefficients of induction is one of the most difficult to carry out with accuracy; and it is doubtless most desirable that we should be able to compare such quantities much more accurately than has been hitherto possible. The usual methods, as far as their general arrangement is concerned, are in all probability not capable of improvement; but, as carried out, there is one modification applicable to all of them, by which the sensitiveness can be vastly increased. This modification is in the use made of the galvanometer. Hitherto it has been usual to observe the throw of the needle due to the passage of the quantity of electricity in one transient current; but if, instead of doing this, we pass through the galvanometer a number of such quantities in rapid succession and (after the manner of Siemens and others) observe the permanent deflection of the needle, we shall find this far greater than any throw attainable by even the most suitable apparatus.

In fact, the permanent deflection due to a certain number of transient currents per second may be compared with the throw of the needle due to one of them, in the following manner:-Let

$\mathrm{H}$ represent the magnetic force acting on the needle,

$G$ the galvanometer-constant,

M the magnetic moment of the needle,

$\tau$ its time of vibration,

* Communicated by the Author. 


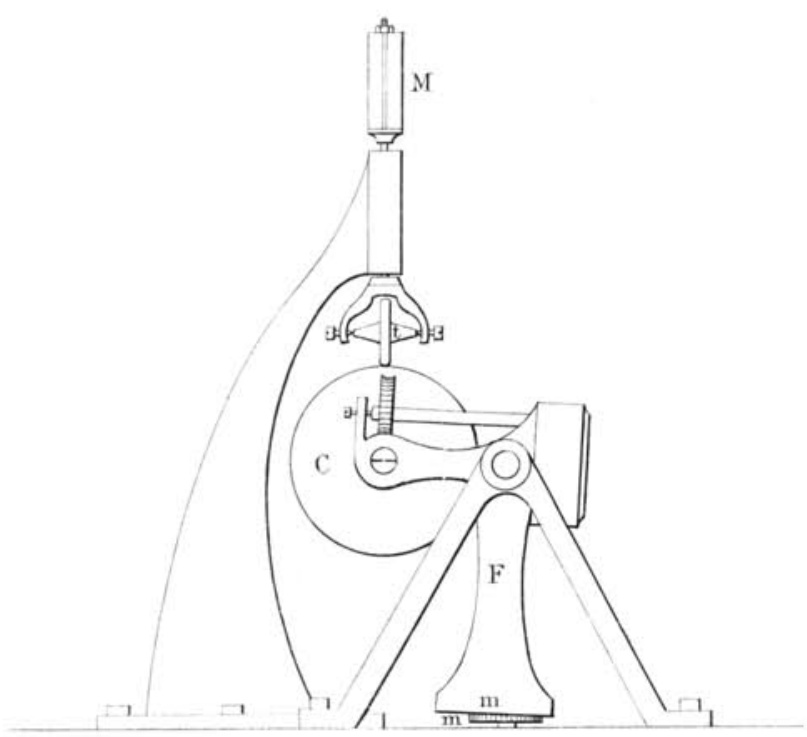

Fig. 8 .
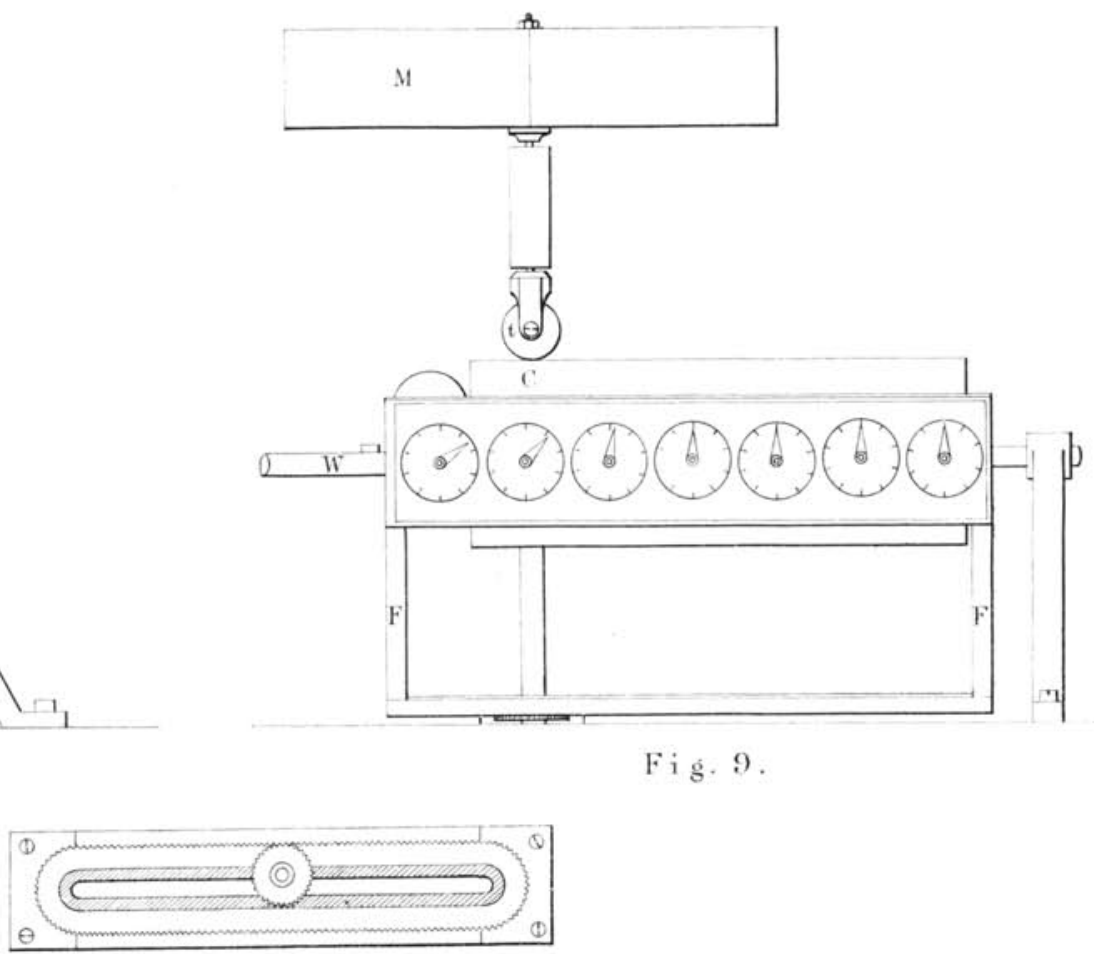

Fig. 10 .

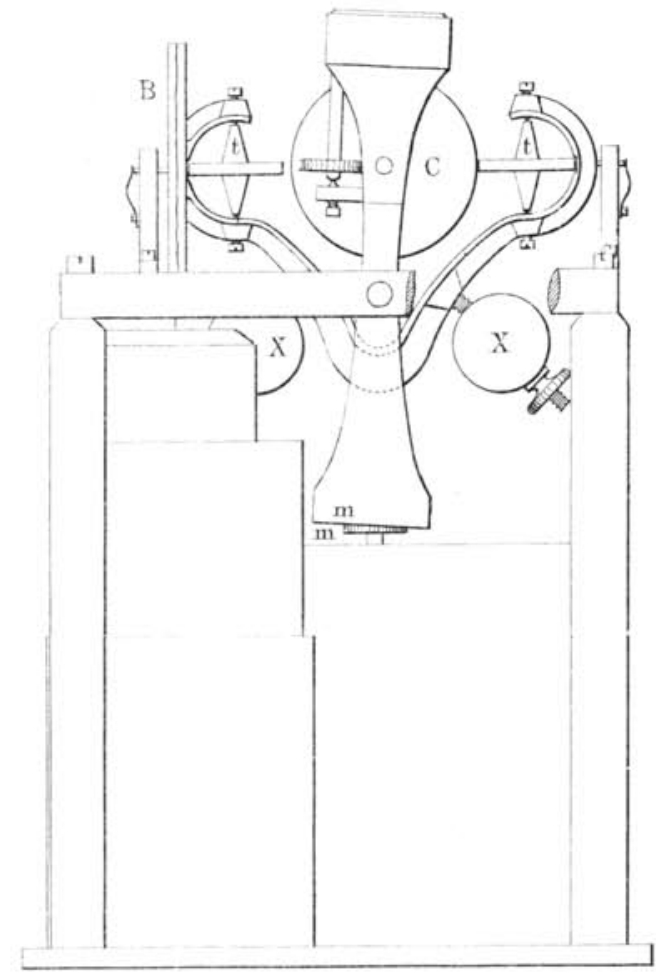

Fig. 11

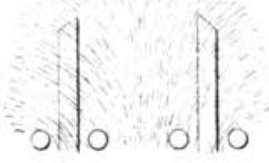

Fiss. 13

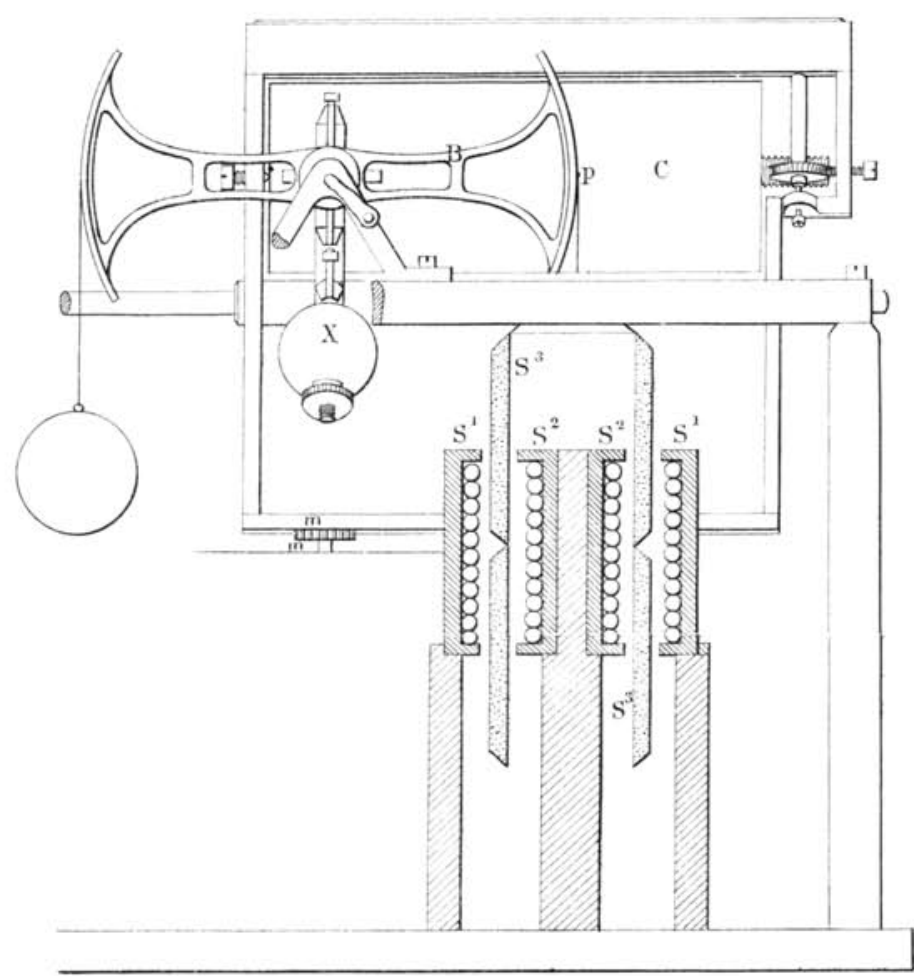

Fi 6.12 .

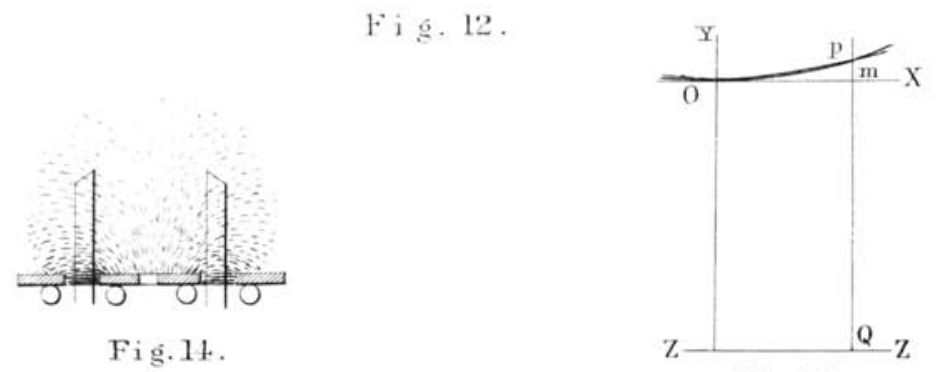

Fig. 15.
Fig. 14 .

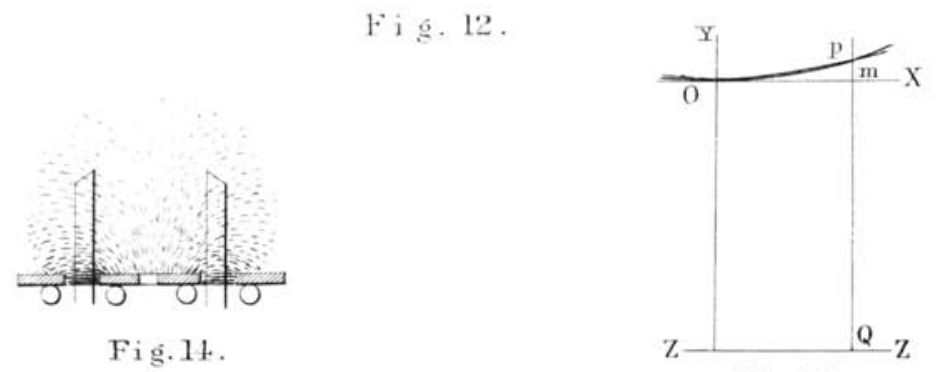

Fi 15.

Fiǵ. 9 . 